12 | 1990

Varia

\title{
Géologie et stratigraphie des Pensées de Pascal
}

\section{Pol Ernst}

\section{OpenEdition}

\section{Journals}

Édition électronique

URL : http://journals.openedition.org/ccibp/639

DOI : $10.4000 /$ ccibp. 639

ISSN : 2493-7460

\section{Éditeur}

Centre international Blaise Pascal

\section{Édition imprimée}

Date de publication : 4 janvier 1990

Pagination : 24-28

ISSN : 0249-6674

\section{Référence électronique}

Pol Ernst, « Géologie et stratigraphie des Pensées de Pascal », Courrier du Centre international Blaise Pascal [En ligne], 12 | 1990, mis en ligne le 08 janvier 2016, consulté le 20 avril 2019. URL : http:// journals.openedition.org/ccibp/639; DOI : 10.4000/ccibp.639

Ce document a été généré automatiquement le 20 avril 2019.

Centre international Blaise Pascal 


\title{
Géologie et stratigraphie des Pensées de Pascal
}

\author{
Pol Ernst
}

\section{NOTE DE L'ÉDITEUR}

Notre ami Pol Ernst a soutenu le 3 février 1990 sa thèse Géologie et Stratigraphie des «Pensées » de Pascal. Le Jury était composé de MM. Irigoin, Professeur au Collège de France, Mesnard, Garapon et Sellier, Professeurs à l'Université Paris IV, et Descotes, Professeur à l'Université Blaise Pascal.

La thèse a reçu la mention Très Honorable à l'unanimité du jury.

Le Courrier du CIBP publie le résumé de ses travaux composé par l'auteur. Il donnera à l'avenir un pareil résumé des thèses pascaliennes arrivées à soutenance.

1 Depuis plus d'un siècle déjà, à diverses reprises, une petite voix s'élève. C'est à peine si on l'a entendue. Peut-être n'y a-t-on guère prêté attention. Aussi bien n'est-il pas inutile de l'écouter.

2 En 1878, Salomon Reinach écrivait : « Il faudrait songer à une édition des Pensées où serait respecté l'ordre chronologique de leur rédaction ». En 1948, Paul-Louis Couchoud reprend cette même invitation : « Les papiers de Pascal ne peuvent être classés dans un ordre satisfaisant, sauf dans l'ordre chronologique.

4 La masse entière ne peut fournir que des ébauches, à classer dans leur ordre successif. »

5 En 1962 - l'année du tricentenaire de la mort de Pascal - Jean Guitton enchaînait : «Je suis d'avis, comme mon vieil ami Couchoud, que l'ordre le meilleur serait un ordre pour ainsi dire géologique, procédant par strates, par couches chronologiques et qui répartirait les Pensées selon leur développement historique ».

6 Peut-on être plus clair? Plus précis? Mais où donc trouver cet «ordre pour ainsi dire géologique » sinon d'abord et exclusivement là où des «strates » avaient existé ? Où elles s'étaient succédées en se superposant les unes sur les autres, c'est-à-dire dans les 
différents paquets de feuilles sur lesquels Pascal avait porté ses réflexions. En d'autres termes : le problème de la chronologie des Pensées et donc celui d'une édition géologique ne peuvent être résolus que grâce à l'identification des "papiers autographes du ms. $9202 »$ »' C'est l'unique recherche qui s'impose, toutes affaires cessantes, et prioritairement. Aussi bien, à la formule adoptée par nos critiques généticiens du XX $\mathrm{XX}^{\mathrm{e}}$ siècle (au commencement était le texte », faut-il en substituer une autre : au commencement était le papier ", plus adaptée aux Pensées. Ce n'est pas par elles qu'il faut commencer, mais par leur support matériel.

Restitution d'un feuillet marqué du filigrane France \& Navarre.

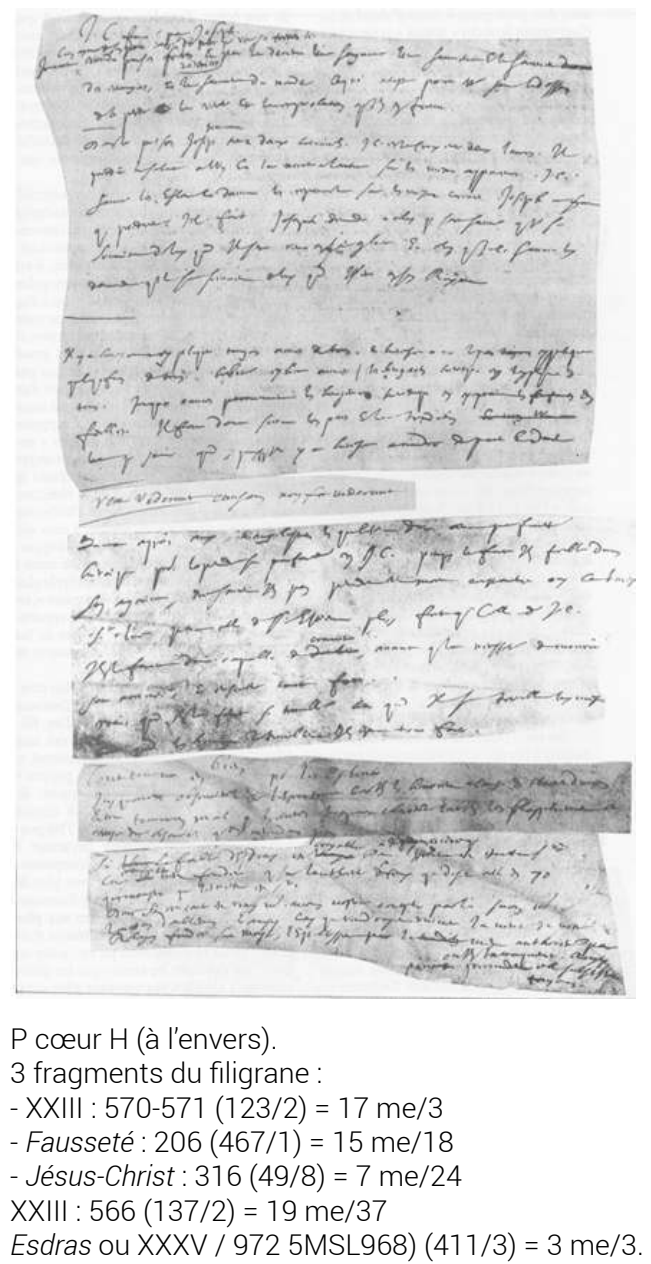

7 Un seul pascalisant néanmoins comprit l'enjeu de cette tâche: Tourneur. Dès 1933, en effet, dans Beauté poétique, il signalait la présence de onze filigranes sur soixante-six «papiers ». Très conscient de l'importance de sa démarche, il en précisait la finalité : « Si la même marque, écrivait-il, se retrouve sur un certain nombre de fragments, il y a fort à parier qu'ils ont été écrits vers la même époque. » Dès 1933 donc, Tourneur assignait à cette tâche son véritable objectif : découvrir des ensembles de textes "probablement contemporains». Dans deux éditions des Pensées («critique» en 1938 et "paléographique » en 1942), le nombre des fragments marqués d'un filigrane avait doublé (quelque 125 « papiers »). Le problème de l'identification des " papiers », s'il avait progressé, était loin d'être résolu : jamais Tourneur n'a proposé une ébauche de synthèse de ses découvertes. Sans aucun doute était-ce impossible parce que prématuré. 
8 Un autre problème non moins important d'ailleurs, occupe les pascalisants qui ont pris conscience de l'ordre de la Copie (ou mieux des Copies). Les éditions objectives prenant en compte cette donnée nouvelle, vont se succéder. Lafuma (1951), puis Ph. Sellier (1976) enfin M. Le Guern (1977). Faut-il le dire? Aucune de ces trois éditions ne fait la moindre allusion aux filigranes. Un problème - celui d'une édition objective - avait enterré celui de l'identification des "papiers». Bel et bien enterré, semble-t-il, puisque l'édition Tourneur-Anzieu elle-même (en 1960) n'avait daigné reprendre aucune des annotations relatives aux filigranes signalés par Tourneur ! «La nature de l'homme n'est pas d'aller toujours. Elle a ses allées et venues ». Sic transit...

Il n'en reste pas moins vrai que les efforts de Tourneur méritaient d'être poursuivis : si son intuition initiale ne pouvait être erronée, sans doute convenait-il d'analyser les raisons de son échec. Peut-être apparaîtrait-il que l'objectif avait été trop restreint (aux seuls filigranes) et que les moyens susceptibles d'aboutir à des résultats décisifs n'avaient pas été envisagés. Peut-être aussi découvririons nous que la rigueur minutieuse et l'observation répétée des originaux autographes avaient fait défaut à celui qui avait déchiffré avec tant de bonheur les fragments eux-mêmes.

Commençons donc par préciser l'objectif. Ne s'agit-il pas de restituer dans leur intégrité primitive des «ensembles de textes contemporains" portés par plusieurs «strates matérielles homogènes »? S'il en est ainsi, il est indispensable d'utiliser tous les moyens grâce auxquels les différentes strates matérielles primitives pourront être restituées dans toute la mesure du possible. En d'autres termes, il est indispensable d'identifier le plus grand nombre possible de "papiers ", et non pas seulement ceux-là qui seraient marqués d'un filigrane. Le filigrane n'occupe qu'une toute petite surface sur un feuillet : à supposer qu'on fût parvenu à identifier tous les "papiers " marqués d'un filigrane, tout compte fait, on n'aurait pas pour autant résolu le problème de la chronologie de la rédaction des Pensées. Il convient donc de s'intéresser à tous les «papiers ». Et surtout à ceux qui sont vierges de toute marque mais qui appartiennent certainement à telle ou telle strate! Dès lors qu'on aurait réussi à identifier le plus grand nombre possible de ces "papiers ", on aurait découvert à quelle strate ils appartenaient, et on serait donc en mesure de les restituer - chacun d'eux - à leur " paquet de feuilles »!

11 Mais avant d'en arriver là, il fallait commencer par vérifier tous les dires de Tourneur relatifs aux "papiers marqués d'un filigrane", ensuite s'assurer s'il les avait tous identifiés, et correctement. Concrètement, si de 1933 à 1938, le nombre de "papiers " marqués d'un filigrane était passé de soixante-six à cent vingt-cinq, ce dernier chiffre correspondait-il à la réalité ? Ne pouvait-on espérer en identifier davantage ? Mais, pour atteindre cet objectif, n'importait il pas, au préalable d'examiner avec plus de minutie les filigranes relevés par Tourneur, portant une attention particulière aux plus minimes différences qui permettraient d'affirmer qu'un filigrane avait pu en cacher un autre, par exemple, ou encore que les dénominations n'étaient pas vraiment adéquates, en conséquence de quoi il était urgent d'en proposer d'autres? Une connaissance générale (mais précise) des filigranes utilisés à Paris entre les années 1650 et 1660 ne s'avérait-elle pas indispensable, elle aussi ?

12 Cette dernière recherche devait révéler les principaux défauts de l'enquête menée par Tourneur : 


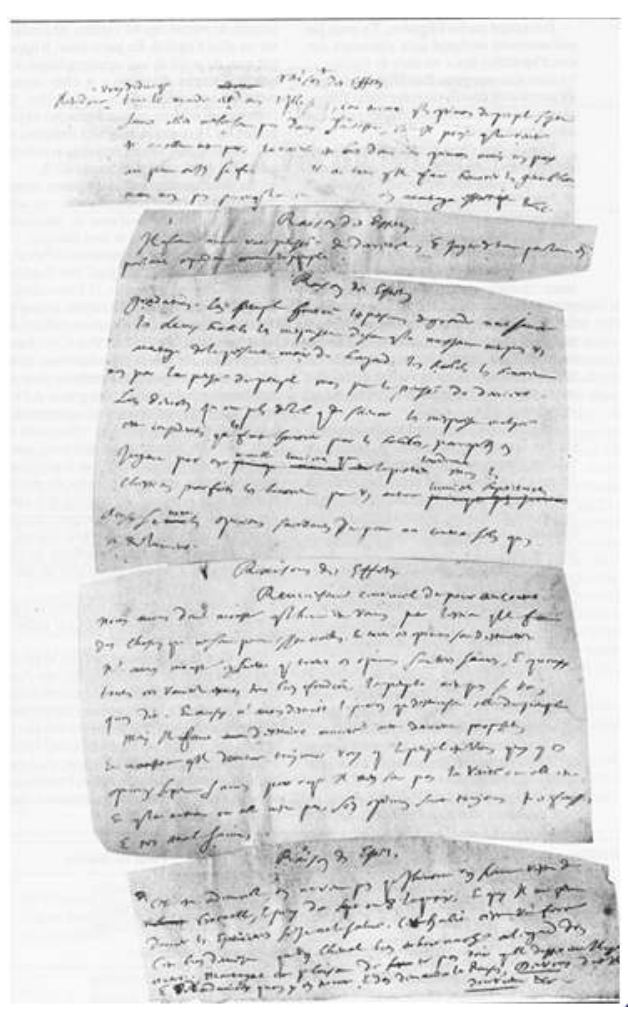

I cour $\mathrm{C}$ Ecartement : 22/23 mm.

Effets : $92(231 / 3)=11 \mathrm{me} / 21$

$91(231 / 5)=12 \mathrm{me} / 21$

$90(231 / 2)=13 \mathrm{me} / 21$

$93(231 / 4)=10 \mathrm{me} / 21$

$89(231 / 1)=14 \mathrm{me} / 21$

Préoccupé par les filigranes, il n'avait pas suffisamment envisagé qu'il convenait surtout d'identifier des "variétés de feuilles »: les unes sont marquées d'un filigrane unique, les autres d'un double filigrane (deux filigranes étaient nécessairement couplés parce que complémentaires).

Ensuite il ne s'était point intéressé du tout aux formats - donnée matérielle capitale non moins que celle des différentes variétés de feuilles. Certes un format identique pouvait être commun à plusieurs variétés, mais certains formats étaient spécifiques !

Enfin il avait totalement négligé de s'intéresser à l'écartement qui sépare les pontuseaux : donnée matérielle histologique indispensable pour identifier les "papiers »! Certes cet écartement peut être commun à plusieurs variétés de feuilles, mais il arrive aussi qu'il est spécifique, auquel cas il est décisif, pour identifier la variété de feuilles sur laquelle Pascal avait prélevé tel ou tel « papier ».

Dès lors que toutes ces données matérielles (le format, le filigrane, la variété de feuilles, l'écartement des pontuseaux) auront été prises en compte, on accordera enfin un intérêt tout particulier à l'indice de fréquence non moins révélateur : ne nous apprend-il pas le nombre exact de feuilles que compte chaque paquet?

17 Telles sont, en résumé, les recherches qu'il a fallu mener pour identifier le plus grand nombre possible de « papiers » et restituer " dans leur intégrité originelle » les différents paquets de feuilles homogènes présentes dans le ms. 9202. 
